\title{
UNMANNED UNDERWATER VEHICLE FOR MONITORING AQUATIC ECOSYSTEM
}

\author{
Abhishek A Nandyal, Adithya D M, Karthik K, Manikantan G \\ Dept of ECE, KSIT \\ Bangalore \\ Dr. P.N. Sudha \\ HOD, Dept of ECE, KSIT \\ Bangalore
}

\begin{abstract}
Collection of Data by Survey and Observation in the actual aquatic environment is indispensable for studies and development. Studies about ocean such as Marine Environment Monitoring, submarine Earthquakes, Ocean life, marine Resource Research are carried out with the help of an underwater vehicle. While the existing underwater vehicles provide a high cost, less effective, inaccurate data processing solution, the problem is addressed by choosing a highly efficient microcontroller PIC18 Microcontroller with its excellent capability for image processing, video streaming and the ability to work based on internet of things. In this paper a low cost and an efficient underwater vehicle is designed and implemented which has the ability to measure parameters like temperature and pressure using sensors.
\end{abstract}

Keywords- Aquatic Ecosystem, Underwater Vehicle, PIC18 Microcontroller.

\section{INTRODUCTION}

The ocean occupies approximately $71 \%$ of the earth surface and still has a lot of unknown parts. Therefore various studies and development about the ocean such as marine environment, submarine earthquake, ocean life, and marine resources research and so on are carried out. The collection of data by survey and observation in the actual sea is indispensable for the studies and the development. Hence there is a need of a low cost, highly efficient underwater vehicle which can serve multiple applications is necessary. Underwater vehicles are the vehicles which work underwater for surveillance and monitoring aquatic ecosystem. They are powerful and complex systems which are capable of performing various underwater tasks.

Thus an underwater vehicle can be considered as the multi body system which performs multiple operations. The dynamic modeling and Simulation of the underwater vehicle is important in the process of design and analysis of the maneuverability of the underwater vehicles. It has become an interesting research area because of their emerging applications. However with the increasing functionalities, the geometric shape and the mechanical design of the vehicle has become more and more complicated. Therefore efficient tools are required to describe detailed and accurate models of the underwater vehicles with complex attached bodies.

With the explosion and combination of the mechanical engineering, electrical engineering and computer science fields, the birth of mechatronics has led to extensive research and development in the area of mobile robotics. Though terrestrial robotics is a field of highly active research and commonly increasing applications, underwater robotic technologies have not grown at the same rate, partially owing to the high costs associated with the development of such robots. Underwater robotic technologies are divided into two basic groups which are Remotely Operated Vehicles (ROVS) and autonomous underwater vehicles (AOVS). Remotely Operated Vehicles (ROVS) are the vehicles which are remotely operated by a highly skilled and trained human operator. Due to rapid failure of remote communication technologies underwater, in order to allow for remote control, these vehicles are physically connected over a tether cable to a remote operation unit.

These vehicles greatly improve efficiency of all kinds of underwater operations by removing the requirement of human presence inside the submersible. Being remotely operated these vehicles can also be deployed in missions who pose a great risk to human life. ROVs gained quick popularity because of their cost- effectiveness and high mission applicability. When such underwater vehicles are made, it is necessary to consider about the following things. 1) Seawater and Water Pressure Environment, 2) Sink, 3) There are n Gas or Battery Charge Stations, 4) Global Positioning System cannot use, 5) Radio waves cannot use.

\section{LITERATURE REVIEW}

In Xiao Bo Tan et al [1] this paper presents an underwater vehicle which has a Robotic fish based on Robotic sensor networks for profiling the aquatic diffusion process where mobile sensors are used for profiling the characteristics of a diffusion process including source location, discharged substance amount, and its evolution over time. Simulation based on real time data traces of GPS localization errors, robotic fish movement, and wireless communication was conducted. An efficient greedy algorithm and a complex 
near optimal radial algorithm were used to solve the limited sensor mobility problem Distributed control algorithms that allow robotics sensors to autonomously plan their motion paths were used. The system has an Aquatic mobile sensor, Robotic fish prototype, GPS, Zigbee antenna and Dissolved oxygen sensor which can be used only on the water surface and it cannot be used under water.

In Lygouras.J.N, et al [2] this paper describes the unmanned underwater remotely operated vehicle (UROV) THETIS, an easy to operate vehicle suitable for exploiting water environments. The vehicle's primary use is to perform underwater observations as well as temperature, $\mathrm{pH} /$ dissolved $\mathrm{O} 2$ and suspended sediment measurements for underwater pollution studies. The THETIS ROV is a remotely controlled submersible, designed to perform water pollution measurements. The system is composed of a ROV, a tethering cable including power supply cable and two optical fibers for communication, and a surface master controlling computer. A slave motherboard (INTEL 80486 microprocessor based) with its appropriate peripherals and plug-in cards as well as the instrumentation and measurement equipment are placed inside the vehicle. Since the vehicle is fed by ordinary AC voltage $(220 \mathrm{~V} / 50 \mathrm{~Hz})$, the necessary power circuits (voltage transformers, rectifiers, regulators, etc.) are embodied as well, in order to create the appropriate voltages needed by each subsystem of the vehicle. THETIS uses four DC motors, rotating specially designed propellers for controlling depth, position on the horizontal plane, and heading of the vehicle. The system is capable of longitudinal, vertical, and lateral motions, as well as turning by means of the four electric thrusters. Heading and depth of the vehicle can be maintained either manually or automatically.

In Xuri Yu, et al [3] the system sampled the region mainly in a saw tooth pattern with horizontal resolution between $\mathrm{B} 120 \mathrm{~m}$ at the middle of the water column and with vertical resolution of $0.1 \mathrm{~m}$. column was characterized by three layers: warm and fresh surface water; cold and salty bottom water; and a transition (pycnocline) layer with sharp vertical temperature and salinity gradients. It has been shown that AUV self-noise and vibration can be reduced to such an extent that meaningful turbulence measurements can be made scientific assessment of fish stocks and to evaluate the extent to which fish avoid ocean-going research vessels as well as to examine spatial variability in the vicinity. The vehicle ODYSSEY is a 2.2 $\mathrm{m}$ long vehicle, which weights between 120 and $160 \mathrm{~kg}$ depending on the sensor and battery configuration employed. The vehicle is capable of ranges in excess of 60 $\mathrm{km}$ at 3 knots, employing its standard silver-zinc battery set. Sensor systems have included: CTD, side scan sonar, 1200 and $300 \mathrm{kHz}$ ADCP, $150 \mathrm{kHz}$ phased array ADCP, eight element acoustic line array, various camera systems including low-light video, and acoustic Doppler velocimeter.

In Wang.W.H et al [4] presents a recently developed low cost UUV prototype at the University of Canterbury was introduced, which was designed specifically for shallow water tasks, especially for inspecting and cleaning sea chests of ships for biosecurity purpose. The main hull of the UUV is made of PVC, with a $400 \mathrm{~mm}$ diameter and $800 \mathrm{~mm}$ length. External frames mount two horizontal propellers, four vertical thrusters, and power is derived from onboard batteries. The maximum thrust force of up to $10 \mathrm{~kg}$ that is provided by the propellers can generate a forward/backward speed of up to $1.4 \mathrm{~m} / \mathrm{s}$ for the $112 \mathrm{~kg}$ UUV. The vertical thrusters provide depth control with a max thrust force of $20 \mathrm{~kg}$. The UUV is equipped with a range of sensors capable of sensing its instantaneous temperature, depth, attitude and surrounding environment. It costs less than US $\$ 10,000$ for a prototype Inspired by torpedoes and submarines a cylindrical hull has been selected. A cylinder has favorable geometry for both pressure (no obvious stress concentrations) and dynamic reasons (minimum drag).

In Matthew Dunbabin et al [5] this paper outlines the development of a new robotic vehicle for underwater monitoring and surveying in highly unstructured environments and presents experimental results illustrating the vehicle's performance. The vehicle represents a new era in AUV design specifically focused at providing a truly low-cost research capability that will progress environmental monitoring through unaided navigation, cooperative robotics, sensor network distribution and data harvesting. A principle aim of the research was to construct a fully autonomous underwater vehicle for less than AUS\$10,000 which requires less than one person/operator per AUV to deploy and operate. Primary tasks that have been identified to be performed autonomously by the vehicle are Video transects, Water quality monitoring and Plume monitoring. The flat thruster is capable of producing in excess of $\pm 8 \mathrm{~N}$ at efficiencies greater than $60 \%$. The three phase motor is self-contained in that it has its own motor driver, propeller and communication hardware and uses the CAN Bus communication protocol to control the motor.

In Charles C. Eriksen et al [6] presents underwater vehicle Sea gliders which are small, reusable autonomous underwater vehicles designed to glide from the ocean surface to a programmed depth and back while measuring temperature, salinity, depth- averaged current, and other quantities along a saw tooth trajectory through the water. Their low hydrodynamic drag and wide pitch control range allows glide slopes in the range 0.2 to 3 . They are designed for missions in range of several thousand kilometers and durations of many months. Sea glider designed to profile up to about $1500 \mathrm{~km}$ of the ocean vertically and $6000 \mathrm{~km}$ horizontally under remote control by choosing a speed and direction in opposition to current averaged over its dive depth, it can profile vertically at a fixed geographic position (the "virtual mooring" mode). Sea glider uses the difference between its dead-reckoned and actual displacements to estimate depth-averaged current. 


\section{A. Project Description}

The model designed is made up of simple PVC pipes which has an approximate diameter of 1 inch assembled with different lengths of PVC pipes. The design uses several U joints and $\mathrm{T}$ joints for assembling the ROV to the required shape. Sealing is done with the use of silicon glue to prevent water entering in to driving system. The movements of the vehicle is controlled with the help of Thrusters which are used for moving the vehicle in forward, backward, up, down, left and right movements of the vehicle. The Thrusters are controlled with the Dc motors driven by L293D driver circuit. The entire vehicle is controlled with help of a credit card size computer PIC18 Microcontroller which is used for interfacing with various sensors like Temperature sensor and Pressure sensor which are used for measuring various physical parameters under water.

\section{B. PROPOSED SYSTEM}

In the proposed system, the initial design starts with selection of material to make the vehicle hull. To make the hull, three easily accessible materials were compared. The first option is to use a section of highly available PVC storm water pipe. The second option involves having a hull made from a composite material, such as carbon fiber or fiber glass. Mandrel spinning of such a hull will allow more freedom in radial dimensions. The process can in fact incorporate a varying radius along the length resulting in a slender, traditional hull. However, this process requires a large amount of design and set up time. A less desirable third option is to use a section of metal pipe, which is prone to corrosion and has a high weight and cost. As a result, the PVC storm water pipe option was selected. The microcontroller used in this project is PIC18 Microcontroller, which is a credit card size computer used in a wide variety of applications. The vehicle is balanced inside water with the help of air holes laid inside the PVC pipes which is placed in the vehicle for maintaining the Zero balance inside water. The vehicle design is such that when there is a chance of system failure when it is under water, the vehicle by itself can reach the upper portion of the water surface through which missing of vehicle is completely impossible. Syringe is also used in the model to collect the water sample.

\section{BLOCK DIAGRAM}

The proposed system has a sensor module which includes a pressure sensor and a temperature sensor which are used to measure the underwater pressure and temperature and display these parameters with the help of a display which is interfaced with a PIC18 Microcontroller which cannot be interfaced with an LCD display directly. The inertial measurement unit has a magnetometer and an accelerometer which are used for finding the direction and speed of the system. The control mechanism is implemented with the help of a thruster which is used for moving in the required direction controlled with the help of a dc motor. The PIC18 Microcontroller is a credit cardsize single board computer which is used as a microcontroller for controlling various operations, has a separate operating system Linux and has built in memory, $\mathrm{ADC}$, camera module etc.

The block diagram of the proposed method is shown below. The block consists of several units like Temperature sensor, Thruster, DC motor, L293D driver, PIC18 Microcontroller and battery for supplying necessary power and PC to control the ROV.

\section{A. Temperature Sensor}

Temperature sensor is used to measure the temperature. It acquires a physical quality temperature and converts it in to a signal suitable for processing -electrical signal using a display unit that is attached to a system. It is a monolithic temperature sensor that generates a modulated serial digital output (PWM) which varies in direct proportion to the temperature of the devices. Here we are also using an analog meter to measure the temperature.

\section{B. Power Supply}

A power supply is a device that supplies electrical energy to one or more electric loads. The term is most commonly applied to devices that convert one form of electrical energy to another, though it may also refer to devices that convert another form of energy (e.g., mechanical, chemical, solar) to electrical energy. The supply voltage is $12 \mathrm{v}$ and $5 \mathrm{v}$. The supply voltage is provided with the use of battery.

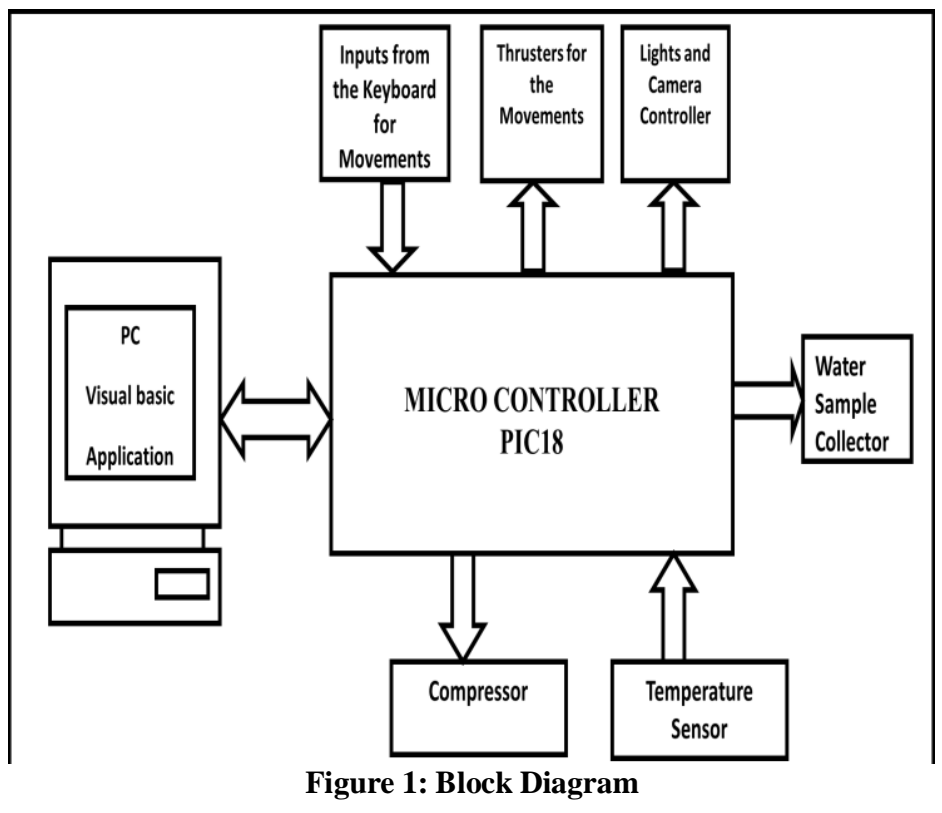

\section{Thruster}

Thruster is used for controlling the movement in the required direction. The two thrusters are used for forward movement and backward movement of the system and the two backward thrusters are used for right and left movements of the system. A thruster is a propulsive device used by water craft for station keeping, attitude control, in the reaction of control system or long duration, low thrust acceleration. 


\section{DC motor}

A dc motor is a machine which converts electrical energy into mechanical energy which can be utilized for controlling the thruster. It works on the principle of Fleming's Left hand rule. The dc motor is driven by the driver circuit LN293D which is used for driving the dc motor. A single driver can drive two de motors .since four dc motors are used two drivers are required to drive four dc motors L293D is a typical Motor driver or Motor Driver IC which allows DC motor to drive on either direction. L293D is a 16-pin IC which can control a set of two DC motors simultaneously in any direction. It has a Dual Hbridge Motor Driver integrated circuit (IC).

\section{E. IC L293D Driver}

This is a motor driver IC that can drive two motor simultaneously. L293D IC is a dual H-bridge motor driver IC. One H-bridge is capable to drive a dc motor in bidirectional. L293D IC is a current enhancing IC as the output from the sensor is not able to drive motors itself so L293D is used for this purpose. L293D is a 16 pin IC having two enables pins which should always be remain high to enable both the H-bridges.

\section{F. Display}

HDMI monitor is not available to use with a PIC18 Microcontroller. It can be very useful to connect remotely using nearby network and laptop. When there is no network available the laptop screen and keyboard can be used with the help of a TTL serial cable, which provides only slow access. A good way to remotely control a PIC18 Microcontroller is to connect to a network a use any of the above method. This will provide access to the terminal by using SSH, with the ability to view desktop using VNC and the ability to run graphical programs directly on the laptop itself. If PIC18 Microcontroller is directly connected to a network it will try to automatically find an IP address by requesting from the router or other device running a DHCP server. However, if there is no DHCP server the pi won't get an IP address.

\section{G. PIC18 Microcontroller}

The PIC18 microcontroller family provides PICmicro® devices in 18- to 80-pin packages that are both socket and software upwardly compatible to the PIC16 family. The PIC18 family includes all the popular peripherals, such as MSSP, ESCI, CCP, flexible 8- and 16-bit timers, PSP, 10bit ADC, WDT, and POR and CAN 2.0B Active for the maximum flexible solution. Most PIC18 devices will provide FLASH program memory in sizes from 8 to 128 Kbytes and data RAM from 256 to 4 Kbytes; operating from 2.0 to 5.5 volts, at speeds from DC to $40 \mathrm{MHz}$ Optimized for high-level languages like ANSI C, the PIC18 family offers a highly flexible solution for complex embedded applications.

\section{WORKING MODEL}

The working model of the system is presented. In this work describes the sensor interfacing portion of the project which is interfaced with PIC18 Microcontroller where communication takes place through the protocols I2C and SPI. The Temperature sensor used is TMP 106 which is a Digital Temperature Sensor has Low voltage operation of 1.4 to $3.6 \mathrm{~V}$ and uses I2C Serial communication Interface. It also has an on chip 12 bit Resolution ADC and Compatible for battery powered applications. The Magnetic Compass used is (HMC6352) which has a Low voltage operation -2.7 to $5.2 \mathrm{v}$ and it uses I2C Serial communication Interface. It has a Field Range - 0.10 to 0.75 gauss (Max Exposed Field-10000 gauss) and can withstand strong magnetic field environment. The Thrusters are controlled with the dc motors driven by 1293d driver.

\section{CONCLUSION}

Thus underwater vehicles play an important role in monitoring, protecting and maintaining the Aquatic Ecosystem which is polluted by leakage in oil spills, accidents caused due to ships etc. A syringe is used for extracting sample for pollution analysis Hence measuring the parameters like temperature, pressure can be used for surveillance in the aquatic ecosystem Temperature sensor and pressure sensor are used for measuring temperature and pressure and display these parameters. Thrusters are used for moving to the required direction controlled with the dc motors driven by driver IC L293D.Accelerometer is used for speed control and Magnetic compass is used for finding the direction. Robotics technologies have found many commercial, scientific, defense and academic applications. As the research in this field increases and other innovations are introduced into the operations of unmanned underwater vehicles (UUVs), UVs will become increasingly more common and highly cost effective.

\section{REFERENCES}

[1] Yu Wang, Ruy Tan, Guoliang Xing, Jianuxun Wang, and Xiaobo Tan. (2014). Profiling Aquatic Diffusion Process Using Robotic Sensor Networks- IEEE Transactions on Mobile Computing, (vol 13, No.4)

[2] Xuri Yu, Tommy Dickey, James, Bellingham, Derek Manov, and Knut Streitlien. (2012). The Application of Autonomous Under Water Vehicles for Inter Disciplinary Measurements in Massachusetts and Cape Cod Bays, (Continental Shelf Research 22)

[3] Nana.L, Singhoff.F, Legrand.J and Marce.L. (2010). Embedded Intelligent Supervision and piloting for Oceanographic AUV

[4] Prof.Sharma.P.B,Prof .Sinha.R.K. (2010). Design and development of the Autonomous Under Water Vehicle 'ZYRA', Robosub Competition Journal Paper (pp. 143$150)$

[5] Charles Eriksen.C, James Osse. T, and Andrew chiodi. M. (2009). Sea glider: A Long -Range Autonoums Underwater Vehicle for Oceanographic Research, IEEE Journal of Oceanic Engineering, ( Vol 26, No.4)

[6] Wang.W.H, Chen. X. Q,and Marbug.A. (2013). Design of Autonomous Under water Vehicle- Japan Agency for Marine-Earth Science and Technology (vol 13,No.2.)

[7] Mathew Dunbaviu , Kane Usher and Peter Corke, 
International Journal of Engineering Applied Sciences and Technology, 2020

Vol. 4, Issue 11, ISSN No. 2455-2143, Pages 493-497

Published Online March 2020 in IJEAST (http://www.ijeast.com)

(2005). A Hybrid AUV Design for Shallow Water Reef Navigation- Proceeding of IEEE International Conference on Automation (Vol 12, No 1.)

[8] Roberto Christi and Anthony Healey.J, (2012).

Adaptive Sliding Mode Control of Autonomous Underwater Vehicle in Dive Plane

[9] www.instructables.com

[10] G.Divya Priya, Mr.I.Harish. (2015) Raspberry Pi Based Underwater Vehicle for Monitoring Underwater Ecosystem, IJETA (Volume 2 Issue 2)

[11] Rossi.L and Krishnamachari (2004). Distributed parameter estimation for monitoring physical phenomenon using diffusion model Comn.Csoc.Sensor Journal.

[12] www.electronicwings.com 